\title{
Residual Stresses in Selective Laser Melted Components of Different Geometries
}

\author{
Mark Reid ${ }^{1, a^{*}}$, Tim Sercombe ${ }^{2, b}$, Anna Paradowska ${ }^{1, c}$, Xiaopeng $\mathrm{Li}^{2, \mathrm{~d}}$ \\ ${ }^{1}$ ANSTO, Australian Centre for Neutron Scattering, Lucas Heights, NSW, Australia \\ ${ }^{2}$ School of Mechanical and Chemical Engineering, The University of Western Australia, Perth, \\ WA, Australia \\ amark.reid@ansto.gov.au, btim.sercombe@uwa.edu.au, canp@ansto.gov.au, \\ dxiaopeng.li@kuleuven.be
}

\section{Keyword: Residual Stress, Neutron Scattering, Selective Laser Melting, Titanium}

\begin{abstract}
As an emerging Additive Manufacturing (AM) technique, Selective Laser Melting (SLM) has found a promising application in biomedical field due to its advantages in fabricating Ti-6Al-4V components with specific and customised geometries. It has been reported that accumulated residual stress as a result of the high heating and cooling rates during SLM and the topological design of the components can largely influence the mechanical and functional properties of the fabricated components. In this study three notched samples were produced by SLM manufacturing. The samples were built using the same laser melting conditions on the same base size. The notches ranged in angle from $60^{\circ}$ to $120^{\circ}$. Residual stresses in the three notched samples were analysed using the state-of-the-art neutron residual strain measurement instrument, Kowari, at the Australian Nuclear Science and Technology Organisation. A combination of gauge volumes were utilised to obtain high precision measurements at the notch tips and general measurements around the tips. This paper reports on the manufacture and measurement of differing residual stresses in the three SLM fabricated notches.
\end{abstract}

\section{Introduction}

As an emerging Additive Manufacturing (AM) technique, Selective Laser Melting (SLM) has found promising applications in a wide spectrum of industries, e.g. aircraft, automotive and biomedical sectors, due to its advantages in fabricating metal components with specific and customised geometries [1]. The process of SLM manufacture is highlighted in Fig 1. Initially an CAD model of the part to be generated is created (a), the model is then segmented into $50 \mu \mathrm{m}$ slices (b). The slice information is then fed to the SLM machine which melts the required pattern into a bed of powder (c). Once the pass is completed the bed is lowered by $50 \mu \mathrm{m}$ and a new layer of powder added (d) and the next melting pass conducted. Finally the completed part is removed from the powder bed (e) and the unused powder is recycled.

An important feature of SLM is that through optimisation of the fabrication parameters (e.g. laser energy, scanning speed, hatch spacing, etc.) and topological design of the components, these SLM fabricated metal components with desired mechanical and functional properties (e.g. ductility, elastic modulus, strength etc.) can be achieved [2]. It is reported that the accumulated residual stress as a result of the high heating and cooling rates during SLM and the topological design of the components can have a significant effect on the mechanical and functional properties of the fabricated components [3-5]. This leads us to deduce that understanding and tailoring the residual stress in SLM components is essential. As part of this understanding the effects of topological features, for a given fabrication method, on the residual stress within a component must be examined. Therefore, to investigate the effects of topography on the residual stress in SLM components, a state-of-art neutron 
scattering facility has been utilised for characterising the residual stress state SLM fabricated metal components of different topographies.

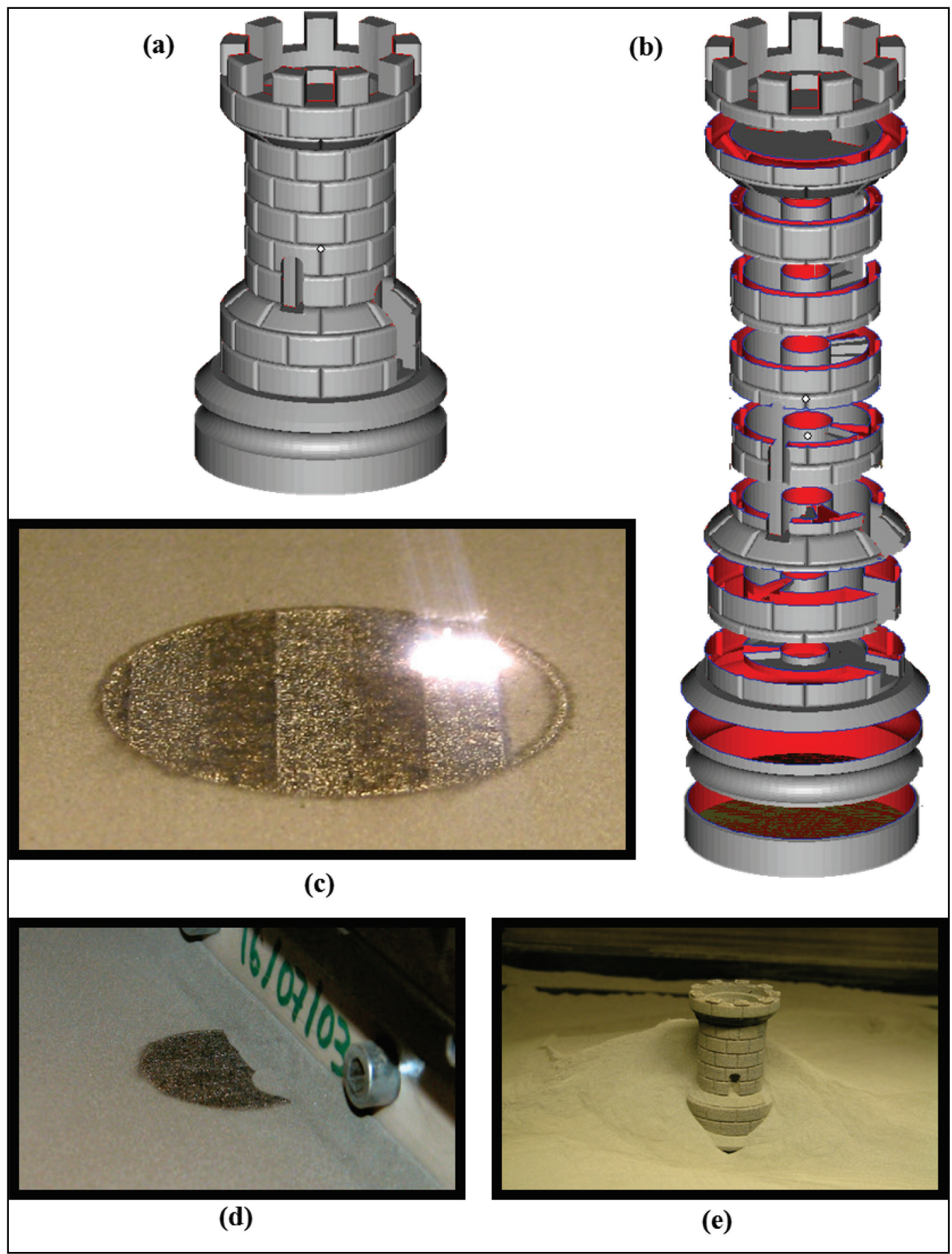

Figure 1. SLM process. (a) CAD model, (b) segmented autocad model, (c) SLM process, (d) new powder layer and (e) removal of final part.

\section{Experimental}

SLM: The Three Dimensional (3D) Printing group in The University of Western Australia produced three notched and one un-notched SLM samples using Ti-6Al-4V powder, size $-53 \mu \mathrm{m}$, supplied by Falcon-tech (China). The geometries of the SLM samples are shown in Fig. 2a. The 
samples were produced in a Realizer SLM-100 machine (ReaLizer GmbH, Germany). The process parameters utilised in manufacturing the components are given in Fig. $2 \mathrm{~b}$.

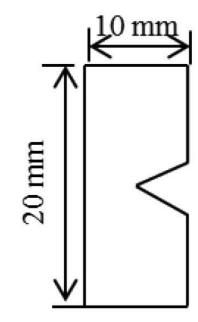

$60^{\circ}$ (a)

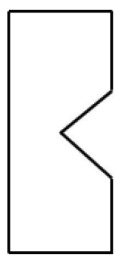

$90^{\circ}$
Thickness of each sample: $5 \mathrm{~mm}$

$120^{\circ}$ (b)

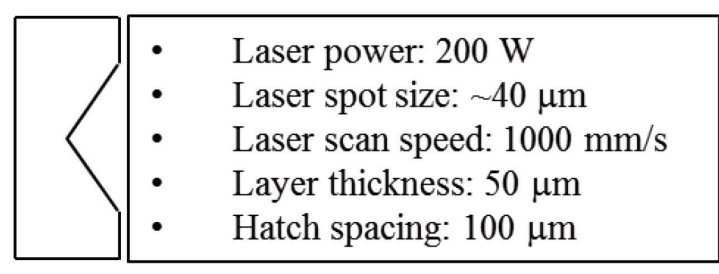

Figure 2. (a) Cross-sections of the SLM fabricated notched Ti-64 components. (b) SLM process parameters

Residual Stress Measurements: The residual strain measurements were performed on the Kowari strain scanning instrument [6] at ANSTO. Because the basic principles of this technique are well known [7-9] only details specific to this measurement will be reported. Neutrons with a monochromatic wavelength are selected from a double focusing monochromator to enable single diffraction peaks to be sampled during strain scanning. Fig. 3b, gives the details of the instrument set-up for the current study and the material properties used in calculating the residual stresses. The sample gauge volume is defined by the primary beam vertical $(\mathrm{pV})$ and horizontal $(\mathrm{pH})$ slits and the receiving slits horizontal width $(\mathrm{sH})$. The measurement locations and measurement direction definitions for the current study and given in Fig. 3a. Two different slit configurations were utilised in this study. The first, $(\mathrm{pV}, \mathrm{pH}, \mathrm{sH}) 1 \times 1 \times 1 \mathrm{~mm}$ was used to examine the bulk of the sample around the notch tips. The second which was used to obtain higher resolution measurement just below the notch tip used a $(\mathrm{pV}, \mathrm{pH}, \mathrm{sH}) 0.5 \times 2 \times 2 \mathrm{~mm}$ gauge volume. The second configuration was only used to measure the longitudinal and transverse directions. As data in the normal direction using the second setup could not be obtained as the gauge volume would have only been partially in the sample, the data for the normal direction from the first setup was extrapolated to provide normal data for the second setup to calculate stresses. Stresses in the longitudinal and transverse direction were calculated based on the assumption that the stress in the normal direction is zero. This allows deconvolution of a stress free reference lattice spacing.

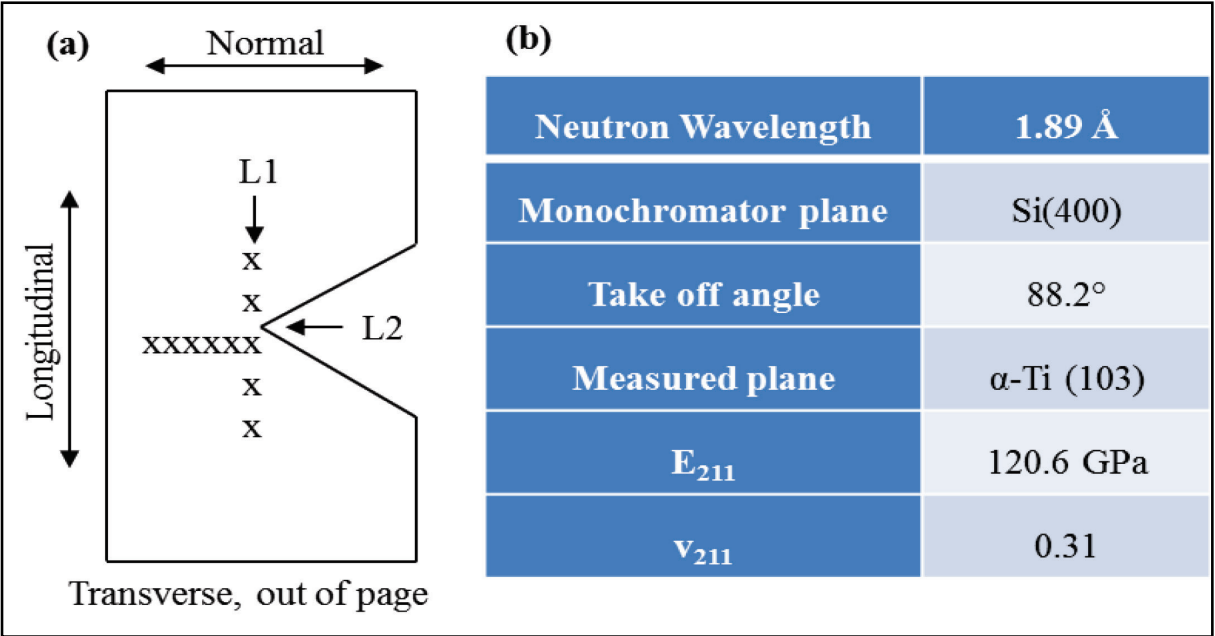

Figure 3. (a) Measurement locations (linel and line 2) and direction definitions for the Kowari experiment. (b) Kowari experimental parameters 


\section{Results}

The results of the residual stress measurements are given in Fig. 4, for the measurement line L1 (Fig 3a) and in Fig. 5, for the measurement line L2 (Fig 3a). The legends for the figures are as follows: Narrow, $60^{\circ}$ notch; Med, $90^{\circ}$ notch; Wide, $120^{\circ}$ notch; Long, longitudinal stresses; Trans, transverse stresses.

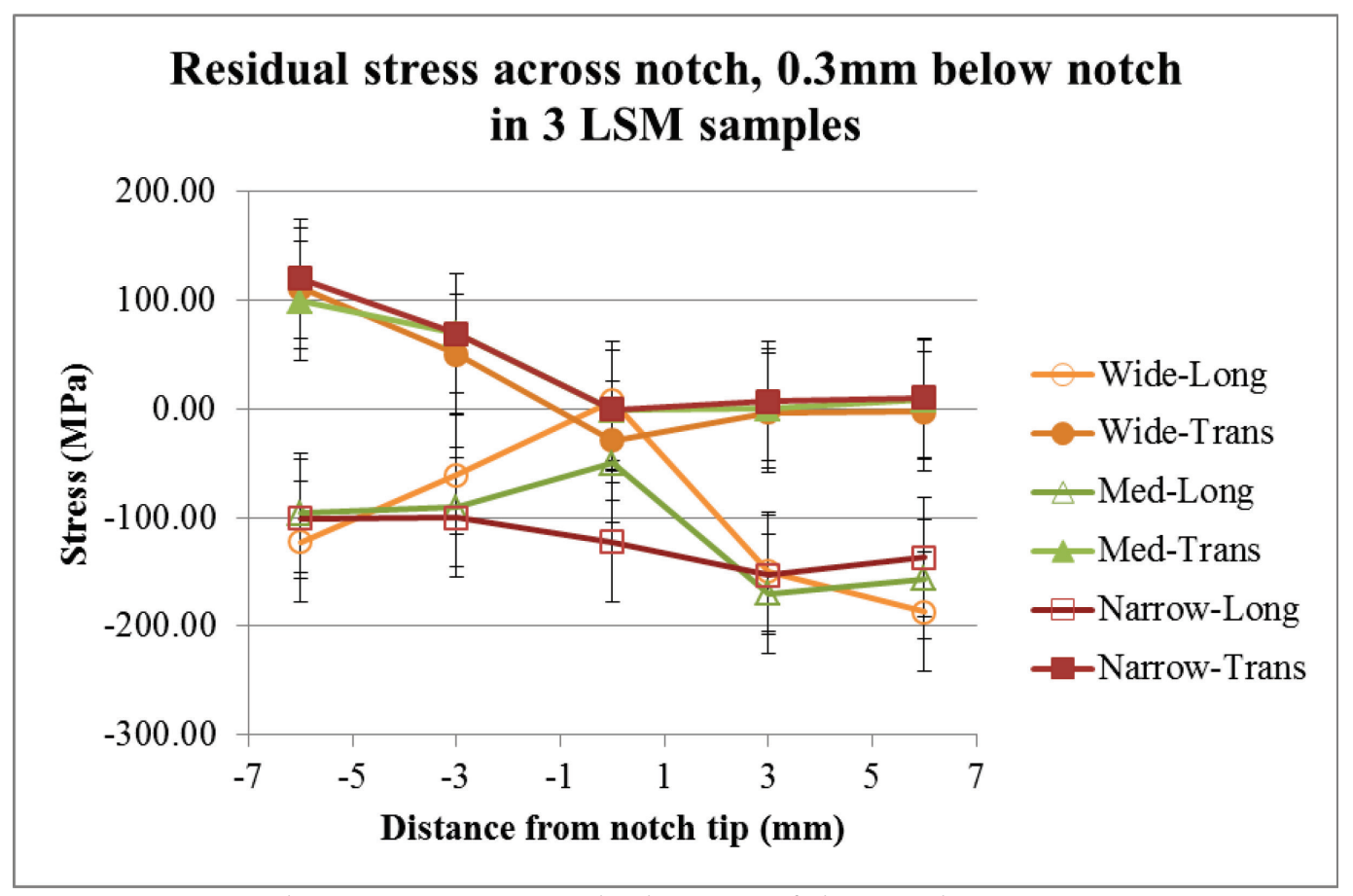

Figure 4. Measurement line L1 traversing the bottom of the notches

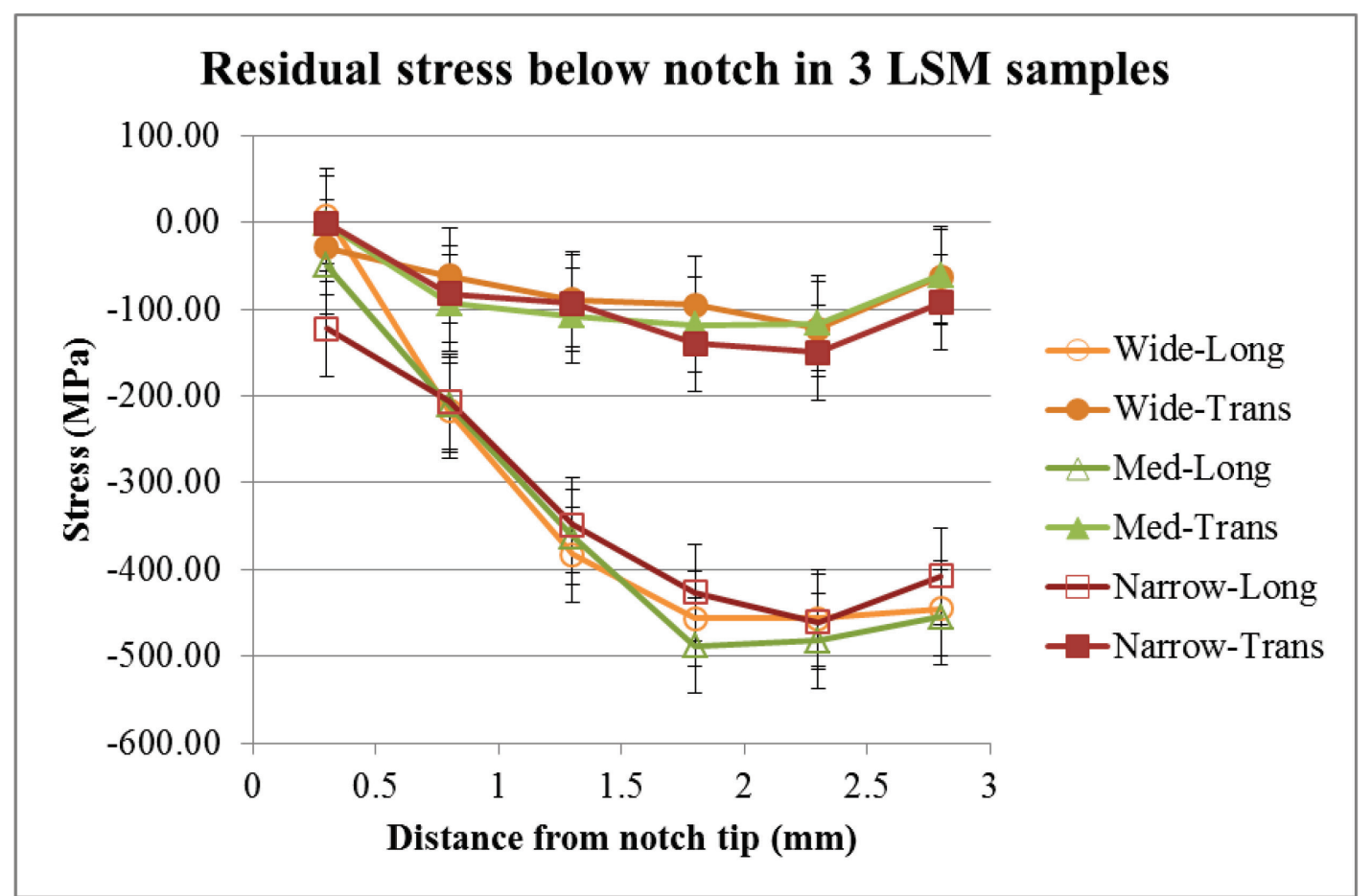

Figure 5. Measurement line L2 starting just below the notches and penetrating into the samples 
Two features are of note in these results. In the case of the residual stresses observed across the base of the notches (Fig. 4) there is a strong asymmetry in the transverse residual stresses in all three of the notched samples. The maximum stress is observed at $6 \mathrm{~mm}$ to one side of the notches, of $\sim 125 \mathrm{MPa}$ in tension which then falls to $\sim 0 \mathrm{MPa}$ below the notch and then remains at this stress out to $6 \mathrm{~mm}$ on the other side of the notch. In the case of the longitudinal stresses there is reasonable symmetry observed. In the case of Fig. 5, where the stresses just below the notches and moving into the samples are shown there is very close agreement with the stresses in the transverse direction. This is generally the case for the longitudinal direction except at the position just below the notch tips. At this location $(0.3 \mathrm{~mm}$ below the notch) there is considerable spread in the observed longitudinal stresses. In the case of the narrow notch $\left(60^{\circ}\right)$ a compressive stress of $\sim 150 \mathrm{MPa}$ is observed, while for the medium notch $\left(90^{\circ}\right)$ the compressive stress is $\sim 50 \mathrm{MPa}$ and for the wide notch $\left(120^{\circ}\right)$ the stress is $\sim 0 \mathrm{MPa}$ (also apparent in Fig. 4).

\section{Discussion}

The results of this study clearly demonstrate that the geometry of SLM manufactured components has an effect on the state of the residual stresses within the components. While this study was to an extent a proof of concept, and as such the authors consider the results somewhat qualitative, some intriguing results have been obtained. Looking at the case of line 2 , below the notch and moving into the sample, the results clearly show that reducing the angle of the notch from $120^{\circ}$ to $60^{\circ}$ has resulted in an increase in the residual compressive stress at the notch. This is not a surprising result as the reduced notch angle will produce greater constraint at the notch tip and hence prevent the relaxation of any stresses developed during the manufacturing process. Different notches would also induce different thermal histories around the notches in different samples. This probably also contributes to the observed difference in residual stresses in samples with different notches. It should be noted that it would be expected the stresses will turn to tensile further into the samples (see $[3,10]$ for example), however these positions were not measured in this experiment due to time constraints.

Turning to line 1, looking at the stresses across the base of the notches, these results are rather surprising. Given in Fig. 6, is the hatch pattern used in forming the components. As each layer is formed the pattern is rotated by $90^{\circ}$. At this stage the authors are unable to account for the observed asymmetry of the transverse residual stresses across the base of the notches. This effect will be the subject of future studies (i.e. samples of the same geometry but different hatch patterns will be examined).

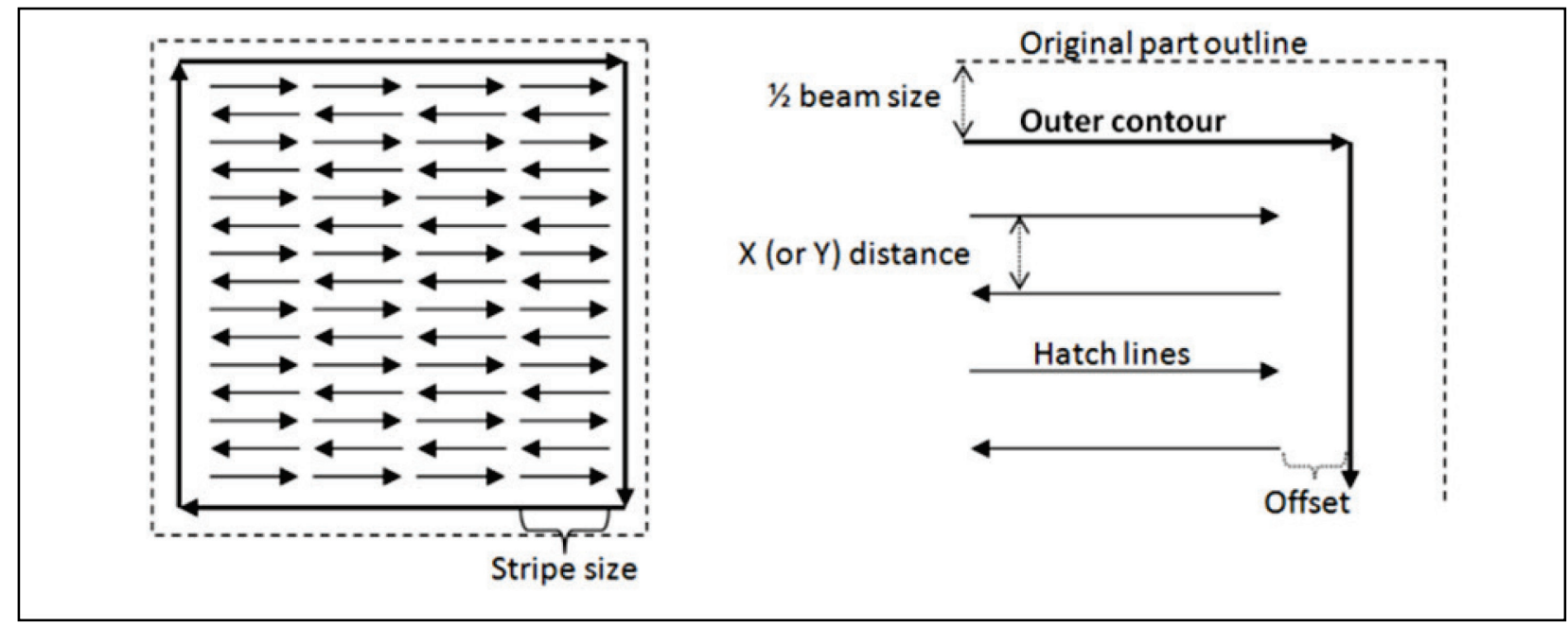

Figure 6. Hatch pattern used in SLM manufacture of the three notched components. 
As noted at the start of the discussion this study is considered a feasibility / proof of concept study which the authors believe has been very successful. Several issues will be addressed in future studies in particular the choice of material for manufacturing the SLM components. Titanium is a strong coherent scatterer of neutrons (contributing to the diffraction peak), however it is also a strong incoherent scatter of neutrons (contributing to noise), thus producing a poor signal to noise ratio in neutron experiments and hence the relatively large error bars reported in Fig. $4 \& 5$. A second issue with titanium alloys is that during high cooling rates martensitic transformation can be induce (not addressed in this report) which can further complicate analysis of residual stresses. For these reasons future studies, which have already been submitted for consideration, will examine more neutron scattering friendly materials (for example austenitic or ferritic stainless steels). Finally the authors envision that the results of this and future studies will contribute real results to efforts to model and thus quantify the effects of geometry and other processing parameters on the residual stresses in SLM components.

\section{Conclusion}

Residual stresses have been measured in 3 SLM manufactured components of different geometries. Three notches, $60^{\circ}, 90^{\circ}$ and $120^{\circ}$ were fabricated from Titanium- $6 \%$ Aluminium- $4 \%$ Vanadium powder. The residual compressive stresses at the notches were $\sim 150,50$ and $0 \mathrm{MPa}$ respectively. This is most likely due to the increased constraint with decreasing notch angle. An unexpected result was that across the notches an asymmetric tensile stress was observed with one side a stress (in all three samples) of $125 \mathrm{MPa}$ observed while the corresponding position on the other side of the notch showed virtually no stresses in the transverse direction. The longitudinal stresses either side on the notches were reasonably symmetric. An explanation for this unexpected stress distribution will be the subject of future studies.

\section{References}

[1] S.M. Giannitelli, D. Accoto, M. Trombetta, A. Rainer, Acta Biomaterialia 10 (2014) 580-594. http://dx.doi.org/10.1016/j.actbio.2013.10.024

[2] B. Vrancken, L. Thijs, J.P. Kruth, J. Van Humbeeck, Acta Materialia 68 (2014) 150-158. http://dx.doi.org/10.1016/j.actamat.2014.01.018

[3] X.P. Li, C.W. Kang, H. Huang, T.B. Sercombe, Materials \& Design 63 (2014) 407-411. http://dx.doi.org/10.1016/j.matdes.2014.06.022

[4] X.P. Li, M. Roberts, Y.J. Liu, C.W. Kang, H. Huang, T.B. Sercombe, Materials \& Design.

[5] X.P. Li, X.J. Wang, M. Saunders, A. Suvorova, L.C. Zhang, Y.J. Liu, M.H. Fang, Z.H. Huang, T.B. Sercombe, Acta Materialia 95 (2015) 74-82. http://dx.doi.org/10.1016/j.actamat.2015.05.017

[6] O. Kirstein,V. Luzin, Materials Australia, 41 (2008) 52-54.

[7] A. Allen, M.T. Hutchings, C.G. Windsor, C. Andreani, Adv. Phys. 34 (1985) 445-473. http://dx.doi.org/10.1080/00018738500101791

[8] M.T. Hutchings and A.D. Krawitz; NATO ASI Series 216E, Kluwer Academic Publishers, Dordrecht / Boston / London (1992).

[9] H.J Prask, P.C Brand, Neutrons in Research and Industry, Proc. of Int. Conf. on Neutrons in Research and Industry, ed: G. Vourvopoulos, SPIE Proceedings Series, 2867 (1997) 106-115. http://dx.doi.org/10.1117/12.267879

[10] X.P. Li, C. Kong, T. Becker, T. Sercombe, Adv. Eng. Mater (2016) 